\title{
Wirkungen von Rohöl-Emulgatorgemischen auf marine Fischbrut und deren Nährtiere
}

\author{
Harald Rosenthal und Wilfried Gunkel \\ Biologische Anstalt Helgoland, Zentrale, Hamburg, und Meeresstation, Helgoland
}

\begin{abstract}
Effects of crude oil-emulsifier mixtures on marine fish fry and their food animals. The effects of crude oil-emulsifier mixtures on the larvae of Cluped harengus $\mathrm{L}$. and Agonus cataphractus L. are described. The herring larvae tested had total lengths of 20 to $26 \mathrm{~mm}$; the larvae of Agonus cataphractus were investigated shortly after their first food intake. Samples of wild plankton from daily catches (used as food during rearing experiments) were also tested. The different dilutions were obtained from the following initial mixture: $20 \mathrm{ml}$ emulsifier "Moltoclar", $80 \mathrm{ml}$ Irak crude oil, $900 \mathrm{ml}$ stale sea water ( $\sim 32 \mathrm{~S} \%$ ). Dilutions with the following content of the emulsifier were used: $50 \mathrm{mg} / 1,25 \mathrm{mg} / 1,5 \mathrm{mg} / \mathrm{l}$, $2.5 \mathrm{mg} / 1,0.5 \mathrm{mg} / 1,50 \mu \mathrm{g} / 1,5 \mu \mathrm{g} / 1,0.5 \mu \mathrm{g} / \mathrm{l}$. Lethal concentrations ranged from 2.5 to $5.0 \mathrm{mg} / 1$. Sublethal damages were clearly ascertained down to a concentration of $0.5 \mathrm{mg} / 1$. Irak crude oil alone did not cause damage to herring larvae during the observation period of 4 days.
\end{abstract}

\section{EINLEITUNG}

Zur Zeit des Tankerunglücks der „Anne Mildred Brøvig “ wurden auf Helgoland Aufzuchtversuche an Larven verschiedener Meeresfische, insbesondere des Herings, durchgeführt. So ergab sich die Möglichkeit, die Wirkung von Emulgatoren, die in größerem Maße zur Ölbekämpfung auf See eingesetzt worden waren, auch auf Fischlarven zu untersuchen. Die Reaktionen der Fischbrut auf die versprühten Emulgatoren erschienen schon deshalb von großem Interesse, weil der Tanker am Rande eines Schollenlaichgebietes gesunken war und gerade zu diesem Zeitpunkt Fischbrut aufkam.

Umfangreiche Untersuchungen über die Eignung von Emulgatoren für die Beseitigung von Öl wurden von Hellmann, Klein \& Knöpp (1966) und Hellmann (1967) durchgeführt. Die Toxizität verschiedener Olbekämpfungsmittel untersuchten KüHL \& MANN (1967) eingehend an verschiedenen See- und Süßwassertieren.

\section{METHODEN}

Untersucht wurden die Larven von Clupea harengus und Agonus cataphractus sowie das aus Netzfängen ( $75 \mu$ und $150 \mu$ Maschenweite) stammende Futterplankton, das von uns für die Aufzuchtversuche verwandt wurde. Die Tiere wurden in Boveri- 
schalen (400 $\mathrm{ml}$ Seewasser) eingesetzt und bei $10^{\circ} \pm 0,1^{\circ} \mathrm{C}$ gehalten. Die Kulturschalen wurden nicht belüftet. Nach einer etwa 2- bis 3stündigen Anpassungszeit wurden jeweils verschiedene Verdünnungen eines Erdöl-Emulgatorgemisches zugegeben.

Als Ausgangsgemisch diente ein Ansatz von $20 \mathrm{ml}$ Emulgator "Moltoclar" und $80 \mathrm{ml}$ Irak-Rohöl in $900 \mathrm{ml}$ gealtertem Seewasser $(\mathrm{S} \sim 32 \%$ ). Zur innigen Vermischung wurde längere Zeit intensiv geschüttelt. Wenn der Anteil Moltoclar auch nicht genügte, um eine stabile Emulgierung des Öles zu erreichen, war es doch möglich, einwandfreie Verdünnungen vorzunehmen. Die Endkonzentrationen des Emulgators betrugen in den Kulturschalen: $50 \mathrm{mg} / 1 ; 25 \mathrm{mg} / 1 ; 5 \mathrm{mg} / 1 ; 2,5 \mathrm{mg} / 1 ; 0,5 \mathrm{mg} / 1 ; 50 \mu \mathrm{g} / 1$; $5 \mu \mathrm{g} / 1 ; 0,5 \mu \mathrm{g} / 1$. Das spezifische Gewicht von Seewasser, Rohöl und Emulgator wurde nicht berücksichtigt, sondern der Einfachheit halber $1 \mathrm{ml}$ gleich $1 \mathrm{~g}$ gesetzt, so daß die Gewichtsangaben nicht ganz exakt sind.

\section{ERGEBNISSE}

Die Wirkungen der verschiedenen Konzentrationen des Erdöl-Emulgatorgemisches wurden durch fortlaufende Beobachtung der Tiere verfolgt. Die Beurteilung der Beeinflussung erfolgte subjektiv durch den Beobachter als Effektbeschreibung zu verschiedenen Zeiten, so daß die Zunahme der Schädigungen zeitlich nicht unbedingt linear erfaßt wurde. Der Grad der Beeinflussung wurde in 6 Stufen unterschieden: $0=$ unbeeinflußt, $1=$ kaum (leicht verminderte Schwimmaktivität gegenüber den Kontrolltieren), $2=$ schwach (deutliche Beeinflussung der Schwimmaktivität), $3=$ mäßig stark (gestörtes Schwimmverhalten, keine Beutereaktionen), $4=$ sehr stark (zeitweise Störung der Gleichgewichtslage, zeitweise inaktiv, gelegentlich hektische Fluchtbewegungen), $5=$ etwa $50 \%$ ständig inaktiv am Boden der Kulturschale, $6=$ Letalitätsgrenze (Aufhören aller Reaktionen, mindestens $50 \%$ tot).

\section{Versuche an Heringslarven}

Die für die Versuche ausgewählten Tiere (Totallänge etwa 20 bis $26 \mathrm{~mm}$ ) hatten am Morgen des Versuchstages noch reichlich Nahrung aufgenommen. In jeder Kulturschale wurden 4 bis 6 Larven gehältert. In den hohen Konzentrationsstufen $(50 \mathrm{mg} / 1$ und $25 \mathrm{mg} / \mathrm{l}$ ) trat bereits nach etwa 2 bis 3 Stunden der Tod ein. Das Ausmaß der Schädigungen war auch deutlich von der Einwirkungsdauer abhängig. Während bei einer Konzentration von $5 \mathrm{mg} / 1$ die Letalitätsgrenze nach etwa 43 Stunden erreicht war, überlebten bei $2,5 \mathrm{mg} / 1$ die Tiere noch mehr als 70 Stunden. Die Ergebnisse sind in Abbildung 1 zusammengestellt.

Die Beeinträchtigung der Schwimmaktivität äußerte sich in den hohen Konzentrationsstufen anfangs besonders deutlich durch kurze Fluchtreaktionen und zittrigen Brustflossenschlag. Nach einiger Zeit erlahmten die Schwimmbewegungen, und die Gleichgewichtslage war gestört. Neben Drehbewegungen traten zeitweise auch noch normale Schwimmbewegungen auf. Ähnliche Beobachtungen machten KüHL \& MaNN (1967) an verschiedenen Tieren. 
In einem gesonderten Versuch wurde eine Kulturschale mit einer dicken Olschicht versehen und kein Emulgator zugesetzt. Obwohl das OOl die Wasseroberfläche völlig abdeckte, konnte selbst nach 4 Tagen keine Beeinflussung der Larven beobachtet werden.

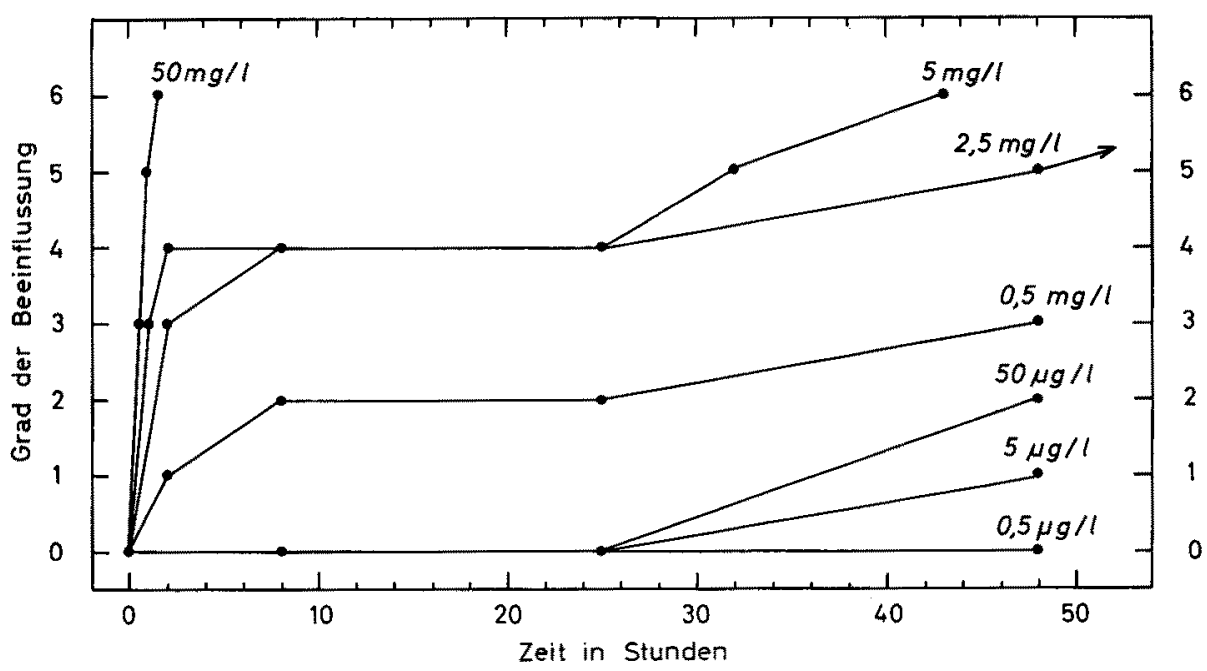

Abb. 1: Einwirkung des Rohöl-Emulgatorgemisches auf Heringslarven $(20-26 \mathrm{~mm}$ Totallänge) in Abhängigkeit von der Einwirkungsdauer und der Konzentration des Emulsionsbildners, Abszisse: Beobachtungszeit in Stunden. Ordinate: Grad der Beeinflussung; $0=$ unbeeinflußt, $1=$ kaum, $2=$ schwach, $3=$ mäßig stark, $4=$ sehr stark, $5=50 \%$ inaktiv, $6=$ Letalitätsgrenze. (Weitere Erläuterungen siehe Text)

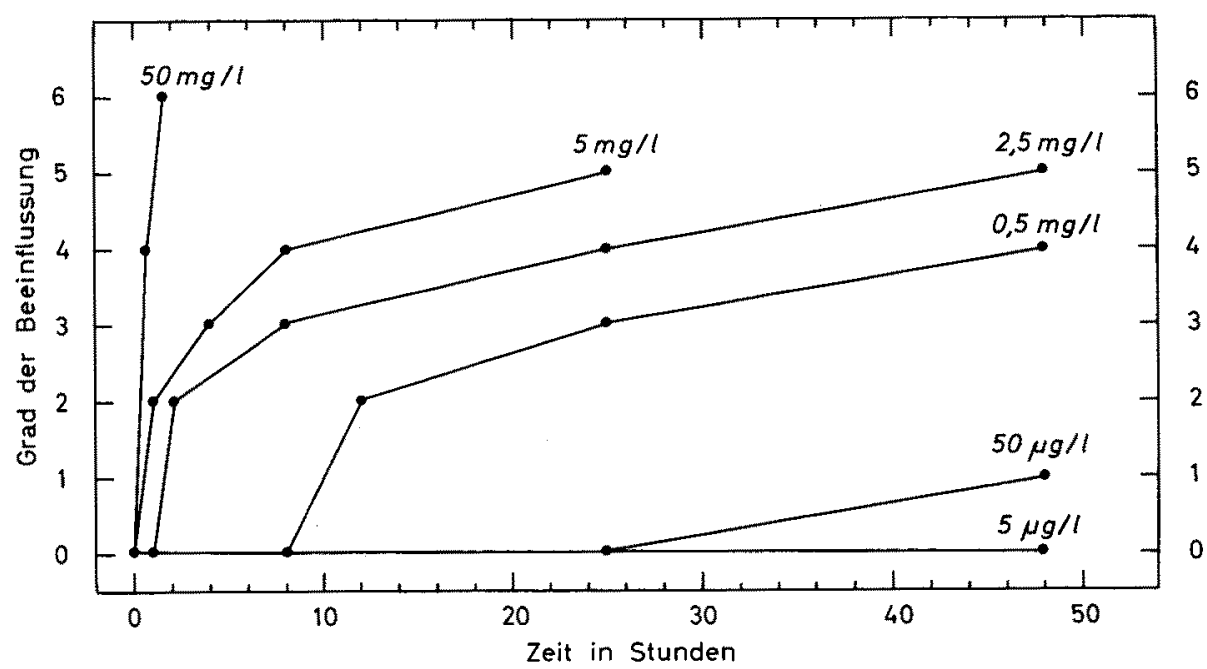

Abb. 2: Einwirkung des Rohöl-Emulgatorgemisches auf die Larven von Agonus cataphractus zur Zeit der ersten Nahrungsaufnahme in Abhängigkeit von der Einwirkungsdauer und der Konzentration des Emulsionsbildners. (Erläuterungen siehe Legende zu Abbildung 1) 


\section{Versuche an $A g$ onus-Larven}

Die Tiere stammten aus Planktonnetzfängen und kamen im Aquarium zur ersten Nahrungsaufnahme. Grundsätzlich verhielten sie sich ähnlich wie die Heringslarven. Die Beobachtungswerte sind in Abbildung 2 zusammengestellt.

\section{Versuche mit Wildplankton}

Das Plankton wurde in gleicher Weise wie die Fischlarven den verschiedenen Konzentrationen des Gemisches O1-Emulsionsbildner ausgesetzt. Es handelte sich um ein Mischplankton von sehr uneinheitlicher Zusammensetzung (Calanus, Pseudocala-

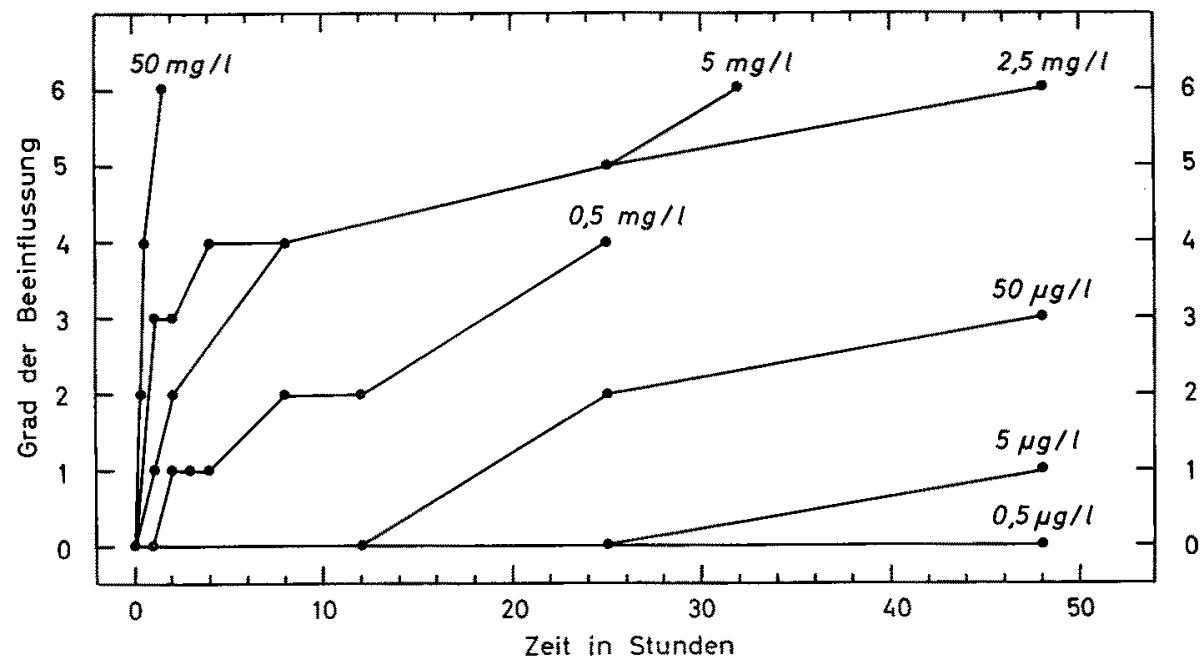

Abb. 3: Einwirkung des Rohöl-Emulgatorgemisches auf Wildplankton in Abhängigkeit von der Einwirkungsdauer und der Konzentration des Emulsionsbildners (Erläuterungen siehe Legende zu Abbildung 1)

nus, Acartia, Temora, Copepoden-Nauplien, Balaniden-Larven, Rotatorien etc.). Die Beeinflussung wurde daher nur pauschal beurteilt. Generell hatte man den Eindruck, $\mathrm{da} B$ die Nauplien-Stadien und Rotatorien empfindlicher reagierten als adulte Copepoden. Abbildung 3 zeigt die Beobachtungswerte.

\section{DISKUSSION}

Die vorliegenden Beobachtungen zeigen, daß das Rohöl-Emulgatorgemisch gegenüber den Testorganismen schon in sehr geringen Konzentrationen subletale Schädigungen hervorruft. Die Ergebnisse über die Letalitätsgrenzen stimmen in den Größenord- 
nungen mit den Befunden von KüHL \& MANN (1967) überein, obwohl sie auf Grund unterschiedlicher Versuchsbedingungen nicht direkt vergleichbar sind. Wir fragen uns natürlich, welche realen Aussagen diese Beobachtungen zulassen. Dabei muß man zunächst feststellen, welche Kriterien schädigende Auswirkungen auf eine Fischbrutpopulation anzeigen können. Neben der Einwirkungsdauer ist das Alter der Larven zum Zeitpunkt der Einwirkung bedeutsam. Fällt dieser mit dem Zeitpunkt der ersten Nahrungsaufnahme zusammen, so kommen bei Einwirkung subletaler Emulgatorkonzentrationen generell zwei Schädigungsmöglichkeiten innerhalb der betreffenden Lebensgemeinschaft in Betracht: (1) Schädigung des Verhaltens der Larven und damit Verlust der Fähigkeit, ausreichend Nahrung zu erjagen und (2) Ausfall der Nährtiere und damit ebenfalls Verhungern des betroffenen T'eiles einer Fischbrutpopulation.

Wie die Versuche gezeigt haben, ist schon nach kurzer Einwirkungszeit auch bei geringen Konzentrationen eine deutliche Störung des Schwimmverhaltens zu beobachten, obwohl die Larven bei Versuchsbeginn in guter Kondition waren und bereits den kritischen Zeitpunkt der ersten Nahrungsaufnahme überschritten hatten. Es ist damit zu rechnen, daß die jüngeren Larvenstadien (insbesondere gegen Ende der Dottersackphase) empfindlicher reagieren.

Zur Bekämpfung der durch die "Anne Mildred Brøvig“ hervorgerufenen Verölung wurden insgesamt 740001 an Emulgatoren versprïht. Das heißt, daß bei Annahme einer gleichmäßigen Verdünnung mit Seewasser etwa $148 \times 10^{6} \mathrm{~m}^{3}$ Seewasser soweit vergiftet würden, daß Fischlarven bei mehr als 48 stündiger Einwirkungsdauer deutlich - wenn auch zunächst subletal - beeinflußt würden.

Das Sprühen des Emulgators wurde von mehreren Schiffen ausgeführt und erstreckte sich über eine ganze Reihe von Tagen, so daß die genannte Menge nicht simultan eingebracht wurde. Das Sprühen wurde auch nur bis etwa Windstärke 4 durchgeführt. Andererseits herrschte im Gebiet eine starke Tidenströmung von etwa $0,5 \mathrm{~m} / \mathrm{s}$. Insgesamt würde bei einer Menge von $148 \times 10^{6} \mathrm{~m}^{3}-$ wenn die Durchmischung bis auf den hier etwa $40 \mathrm{~m}$ tiefen Grund vor sich gegangen wäre - ein Streifen von $370 \mathrm{~m}$ Breite und $10 \mathrm{~km}$ Länge vergiftet worden sein. Sicher ist die Vermischung nicht so vor sich gegangen, da nur bei relativ ruhigem Wetter gesprüht werden konnte.

Es wurden damals keine Untersuchungen über die auftretenden Konzentrationen in situ durchgeführt. Es muß auch angenommen werden, daß die Durchmischung ungleichmäßig erfolgte, so daß sich Inseln mit hohen Emulgatorkonzentrationen in Wasser mit relativ niedrigen Emulgatorkonzentrationen befanden. Wir können also keine Angaben und Aussagen über die tatsächliche Geschwindigkeit der Verdünnung machen. Hinzu kommt, daß Fischlarven merkliche Fluchtreaktionen zeigen können; so ist ohne weiteres denkbar, daß Larven, falls eine vergiftete Deckschicht von $1 \mathrm{~m}$ Durchmesser vorliegt, diese durch Fortschwimmen meiden. Unbekannt ist weiterhin, inwieweit sich Larven erholen konnten, nachdem die subletal schädigende Konzentration weiter abfiel. Schließlich wissen wir nicht, in welchen Mengen die besonders toxische Fraktion des Emulgators, der niedrigsiedende organische Anteil des Lösungsmittels, verdunstete.

Quantitative Aussagen über das Ausmaß der Schädigungen durch Emulgatoren beim Tankerunglück der „Anne Mildred Brøvig“ können nicht gemacht werden, obwohl wir gewisse lokale Schädigungen annehmen müssen. Im Falle der Verölung durch die "Torrey Canyon" wurden an der Küste Cornwalls Emulgatoren in bedeutend 
höherem Maße eingesetzt. Hier sind bis zu 90\% der Benthosorganismen vernichtet worden (NeLSON-SmITH 1967).

\section{ZUSAMMENFASSUNG}

1. Es wurde die Einwirkung eines Rohöl-Emulgatorgemisches (Irak-Ol/Moltoclar) auf die Larven von Clupea harengus L. und Agonus cataphractus L. sowie auf Wildplankton untersucht.

2. Bei Emulgatorkonzentrationen von 2,5 bis $5,0 \mathrm{mg} / 1$ war die Letalitätsgrenze erreicht.

3. Subletale Schädigungen ließen sich bis zu einer Konzentration von $0,5 \mathrm{mg} / 1$ deutlich nachweisen.

4. Irak-Rohöl hatte keine schädigenden Wirkungen auf Heringslarven.

\section{ZITIERTE LITERATUR}

Hellmann, H., 1967. Entölung von Gewässeroberflächen - eine Anwendungsmöglichkeit für Tenside? Tenside 74 (11), 352.

- KLEIN, K. \& KNöpp, H., 1966. Untersuchungen über die Eignung von Emulgatoren für die Beseitigung von $\mathrm{O} 1$ auf Gewässern. Dt. gewö̈sserk. Mitt. 10, 29-35; 60-70.

KüHL, H. \& ManN, H., 1967. Die Toxizität verschiedener Olbekämpfungsmittel für See- und Süßwassertiere. Helgoländer wiss. Meeresunters. 16, 321-327.

Nelson-Smith, A., 1967. Oil, emulsifiers and marine life. In: Conservation and the Torrey Canyon. J. Devon Trust Nat. Cons. (Suppl.) 1967 (7), 29-33. 